\title{
nature
}

\section{Following through on Phillips}

Britain's inquiry into the BSE crisis has revealed significant weaknesses in the way the government used scientific advice and established research priorities on a topic of urgent social concern. These require more detailed public scrutiny.

$\mathrm{D}$ id Lord Rothschild get it wrong? Academic research financed by UK government departments is still based on the 'customer/contractor' relationship proposed by Rothschild almost 30 years ago. Last week, as Lord Phillips' public inquiry into the recent crisis over bovine spongiform encephalopathy (BSE) exposed the blurring of scientific and political judgements inside the Ministry of Agriculture, Fisheries and Food (MAFF), some observers were asking if Rothschild made a mistake in inviting just such an overlap.

There is certainly much in the inquiry's report to support this view. At its heart is the finding that public statements by MAFF, and its decisions on research directions, were dominated by a political objective: the need to provide reassurances about the safety of British beef. Evidence given to the Phillips committee reveals the wide impact of this mind-set. For example, the political goal of reassurance seems to have been closely linked to support for the scientific hypothesis that BSE was a variant of scrapie, present in UK sheep for many centuries.

Such evidence might be taken as a warning against ever allowing political agendas to influence scientific priorities. But a different conclusion can also be drawn, that the problem was not the attempt to impose policy-related priorities on research in an area of vital social concern, but rather the misguided nature of those priorities.

In other circumstances, political priorities have shaped the research agenda in a more positive way. The UK government's decision to appoint an AIDS research supremo in the 1980s to review the scientific agenda, compare it with public-health needs, and commission research to fill perceived gaps, was one example. If the BSE research agenda had been controlled by the Department of Health rather than MAFF, with public rather than animal health dominating the agenda, the outcome might have been very different.

In other words, Rothschild got it right, at least up to a point. The failure was one of implementation, rooted in political dynamics. The influence of political muscle is highlighted by the ill-fated attempts by health-department officials in 1990 to persuade MAFF to appoint a single research supremo for BSE, similar to the AIDS post (see page 5). A striking feature of Phillips' description of this episode is the absence within government of any individual with sufficient clout to establish a coherent research policy on BSE, properly informed by the nation's leading scientific experts.

Why this situation arose is a question of pressing national interest, sufficiently pressing to justify a separate inquiry into the linked dynamics of science advice and priority setting. The Royal Society has taken a step in the right direction by promising a quick report on the governance of science. But the Phillips report provides a unique opportunity to go further. A new public inquiry could revisit sensitive topics, from the quality of research in government labs to the ways in which scientific advice is put into practice. It should also address whether BSE research should be handled by the Food Standards Agency, not MAFF. The challenge is daunting, but the need is urgent.

\section{Total eclipse unlikely}

\section{Even as large optical telescopes steal much of the limelight, smaller instruments can retain an important role.}

$\mathrm{T}$ he world of astronomy is entering a new era of large optical telescopes - giant pieces of equipment with primary mirrors measuring eight or more metres across. The light-gathering capacity of these enormous 'photon buckets' gives them inherent advantages: astronomers can observe fainter sources, and therefore address problems that were beyond the capacity of smaller telescopes. They can also observe brighter sources much more quickly, speeding up project execution.

Add to that the fine resolution made possible by combining a large mirror with adaptive-optics techniques, which correct for the distorting effects of atmospheric turbulence, and it is easy to see why the operators and users of small telescopes feel threatened. Money for astronomy comes from a limited pot, and with titanic telescopes consuming an ever-bigger share of it, many smaller observatories are coming under pressure to close (see page 12).

But although some older telescopes will have to go, it would be a mistake to cull the majority. For a start, many projects don't require giant mirrors, and can be efficiently conducted using a modest-sized telescope. And any study requiring repeated observations of the same object can only realistically be done using a small telescope. If the discoverers of extrasolar planets had had to compete for observing slots on one of the 10-metre Keck telescopes, for example, their high-risk endeavour would never have been granted enough time to bear fruit.
There is also a social factor: the shift towards fewer, larger telescopes inevitably places power in the hands of a privileged élite of astronomers. If diversity is strength, then, for the good of the astronomical community, it is important to retain a sufficient number of smaller telescopes to allow independent groups to do their own thing.

But given limited budgets, the users of small telescopes will have to look beyond a 'business as usual' approach. The small telescopes that survive into the era of the giants will be those that best demonstrate their efficiency and scientific productivity. That may mean that telescopes will have to concentrate on focused projects, rather than holding open competitions for observing time. It may also mean that ownership of these telescopes should pass from centralized organizations with relatively high overhead costs to leaner and fitter consortia of universities or research groups.

Finally, astronomers will need to change their attitudes towards the technical specialists who build instrumentation. If small telescopes are to work on focused projects, they will need to be fitted with highly specific instruments, optimized for the task in hand. Currently, those who build detectors and other instruments are sometimes treated as second-class citizens by certain of their colleagues. Their skills should be granted the respect already enjoyed by the builders of instruments in other scientific disciplines such as space science and high-energy physics. 